\title{
Antibiotic-associated hemorrhagic colitis with ischemic change
}
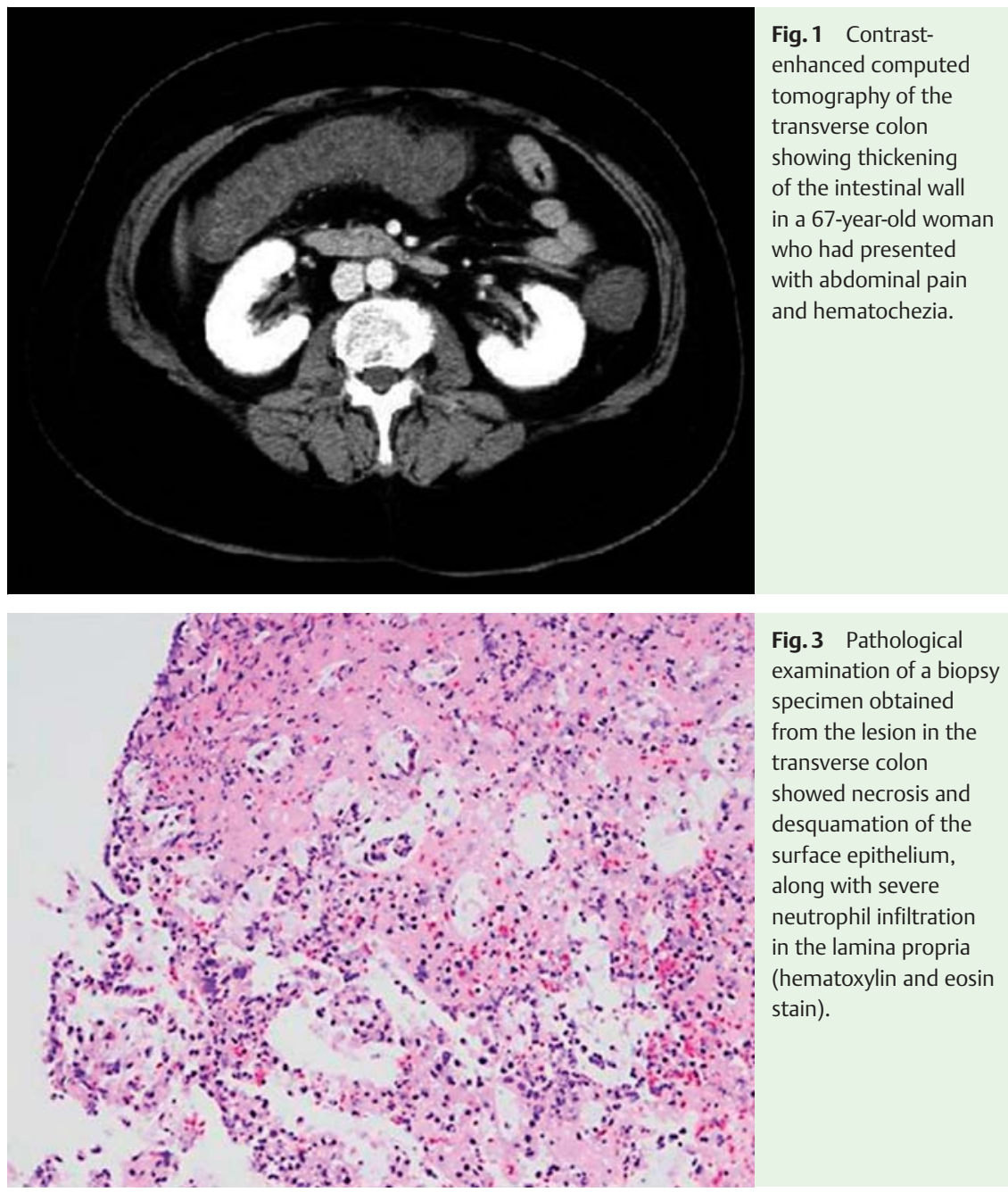

A 67-year-old woman presented with abdominal pain and hematochezia. She had never used non-steroidal anti-inflammatory drugs, but had been started on amoxicillin, clarithromycin, and lansoprazole 3 days earlier as Helicobacter pylori eradication therapy. On admission, white blood cell count was $21600 / \mu \mathrm{L}$ and C-reactive protein level was $2.8 \mathrm{mg} / \mathrm{dL}$. Computed tomography showed thickening of the intestinal wall from the ascending to transverse colon ( Fig. 1). No occlusion of the superior mesenteric artery was found.

We prohibited ingestion and started an intravenous drip without antibiotic. Klebsiella oxytoca was detected in culture of a fecal sample taken on admission, and neg-
Fig. 1 Contrastenhanced computed tomography of the transverse colon showing thickening of the intestinal wall in a 67-year-old woman who had presented with abdominal pain and hematochezia. examination of a biopsy specimen obtained desquamation of the epithelium, neutrophil infiltration (hematoxylin and eosin stain)

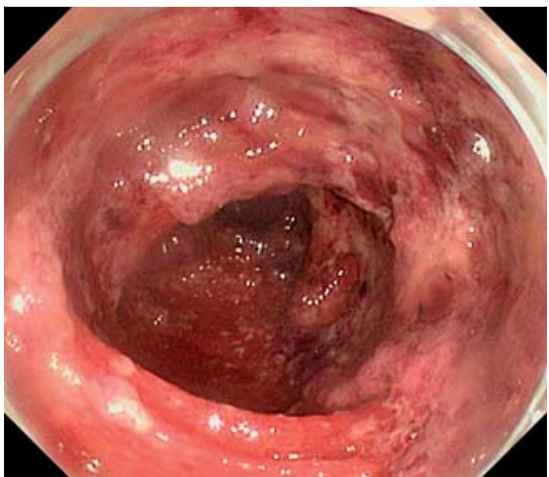

Fig.2 On hospital day 4, colonoscopy of the transverse colon revealed a deep longitudinal ulcer with dark purple color change.

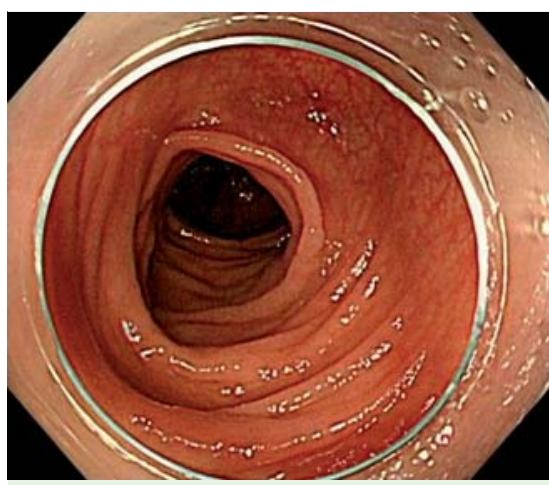

Fig. 4 Follow-up endoscopy of the transverse colon on hospital day 16 showed that the lesion had completely disappeared.

mains unknown. Some reports have suggested $K$. oxytoca as the cause $[1,2]$, but this bacterium has not been consistently isolated [3]. Diffuse mucosal hemorrhage is described as a typical endoscopic finding of AAHC, whereas ulceration is uncommon [3]. Although a variety of histological changes have been reported in AAHC, few reports have described longitudinal ulceration. The major pathological findings seem to be intramucosal hemorrhage with generally little inflammatory cell infiltration $[4,5]$.

Endoscopy_UCTN_Code_CCL_1AD_2AJ

\section{Competing interests: None}


Koichiro Abe, Yu Kawashima, Hitoshi Aoyagi, Satoshi Kimura, Akari Isono, Tadahisa Ebato, Takatsugu Yamamoto, Hiroto Kita, Yasushi Kuyama

Department of Internal Medicine, Teikyo University School of Medicine, Tokyo, Japan

\section{References}

1 Totani T. Acute hemorrhagic enteritis with Klebsiella oxytoca. Nihon Rinsho 1978; 36 : $1308-1309$

2 Högenauer C, Langner C, Beubler E et al. Klebsiella oxytoca as a causative organism of antibiotic-associated hemorrhagic colitis. NEJM 2006; 355: 2418-2426

3 Kishida T, Sato J, Fujimori $S$ et al. An endoscopic study of antibiotic-associated hemorrhagic colitis. Nihon Ika Daigaku Zasshi 1992; 59: 450-456

4 Satake Y, Takahashi Y, Yamamura $M$ et al. Endoscopic and histopathological differences between antibiotic-associated hemorrhagic colitis and ischemic colitis. Gastroenterol Endosc 1985; 27: $344-350$

5 Sakurai Y, Tsuchiya H, Ikegami $F$ et al. Acute right-sided hemorrhagic colitis associated with oral administration of ampicillin. Dig Dis Sci 1979; 24: 910-915

\section{Bibliography}

Dol http://dx.doi.org/

10.1055/s-0034-1390717

Endoscopy 2014; 46: E601-E602

(c) Georg Thieme Verlag KG

Stuttgart · New York

ISSN 0013-726X

\section{Corresponding author}

\section{Koichiro Abe, MD}

Department of Internal Medicine

Teikyo University School of Medicine 2-11-1 Kaga

Itabashi-ku

Tokyo 173-8606

Japan

Fax: +81-3-53751308

abe@med.teikyo-u.ac.jp 